\title{
Actitud, percepción y tolerancia a la violencia en estudiantes de la generación milenio
}

\section{Attitude, perception and tolerance towards violence among Millennial Generation students}

\section{ISSN 2071-8748 \\ E-ISSN 2218-3345 \\ (c) (1) $\$$ \\ BY NC SA}

URI: http://hdl.handle.net/11298/961

DOI: https://doi.org/10.5377/entorno.v0i67.7502

\author{
Hilda Burgos-Ocasio, Ph.D. \\ Ana R. Pinilla-Díaz, Ph.D. \\ Pontificia Universidad Católica de Puerto Rico \\ Escuela Graduada de Trabajo Social Escuela Graduada de Psicología \\ hburgos@pucpr.edu apinilla@pucpr.edu \\ Recibido: 10 de agosto 2018 \\ Aprobado: 30 de enero 2019
}

\section{Resumen}

El presente estudio fue uno de tipo exploratorio, con el propósito de estudiar las diferencias por género de la generación milenio en actitudes, percepción y tolerancia hacia la violencia. Se realizó con una muestra por disponibilidad de 78 estudiantes universitarios, de una universidad pública en Puerto Rico. Se administró una hoja de información sociodemográfica y la Escala de Actitudes, Percepción y Tolerancia a la Violencia (PinillaDíaz y Burgos-Ocasio, 2012). Se analizaron las estadísticas descriptivas para los datos sociodemográficos y las diferencias entre grupos, por género, con t de Student, para las variables dependientes: actitud, percepción y tolerancia a la violencia. En la variable actitud hacia la violencia [ $t(75)=t-3.758, p=.001$.] no se encontraron diferencias significativas entre hombres $(M=53.789$, $D . E .=6.115)$ y mujeres $(M=59.590, D . E .=4.271)$. Esto se reportó también en la variable percepción a la violencia, hombres $(M=79.083, D . E .=23.916)$, y mujeres $(M=88.667, D . E .=19.598)[t(70)=-1.698, p=.097]$. Por otro lado, se encontraron diferencias significativas en los niveles de tolerancia, al compararlos entre hombres

\section{Abstract}

This exploratory study had the purpose of studying the gender differences of the millennium generation in regards to the attitudes, perception and tolerance towards violence. It took into account a sample of 78 university students from a public university in Puerto Rico. A sociodemographic information sheet was administered along with the Escala de Actitudes, Percepción $y$ Tolerancia a la Violencia--Scale of Attitudes, Perception and Tolerance to Violence-- (Pinilla-Díaz and BurgosOcasio, 2012). An analysis of the descriptive statistics for the sociodemographic data and differences between groups, by gender, was carried out using the Student's t-distribution, in relation to the dependent variables: attitude, perception and tolerance to violence. In the variable attitude towards violence $[\mathrm{t}(75)=\mathrm{t}-3.758, \mathrm{p}=$ .001.] there are no significant differences between men $(M=53,789, S D=6,115)$ and women $(M=59,590, S D=$ $4,271)$. This is also reported in the variable perception of violence: men $(M=79,083$, D.E. $=23,916)$, and women $(\mathrm{M}=88,667, \mathrm{D} . \mathrm{E} .=19,598)$ [t $(70)=-1,698, \mathrm{p}=.097]$. On the other hand, significant differences were found in the 
$(M=27.522, D . E .=22.520), \mathrm{y}$ mujeres, $(M=22.580, D . E$. $=3.441)[t(71)=3.796, p=.001]$. La magnitud de las diferencias en promedios fue una grande (eta cuadrado $=.17)$. En otro aspecto, el género femenino presentó diferencia en sus niveles de actitud y tolerancia. Se discuten las implicaciones de la exposición a la violencia, del género y de la generación milenio y la necesidad de desarrollar investigaciones entre estas variables y la desconexión moral.

\section{Palabras clave}

Violencia-estadística, violencia en la educación, violencia contra la mujer, delitos contra la mujer, conducta (psicología), problemas sociales, psicología social.

\section{Introducción}

Estudios recientes sobre la violencia resaltan los efectos y factores de riesgo asociados con esta. Dentro de estos factores se destaca la exposición a la violencia, como un factor que produce desensibilización y tolerancia a esta (Bushman, Calvert, Dredze, Jablonski, Morril, Romer, Newman, Downey, Gottfredson, Masten, Neill y Webster, 2016; Pinilla-Díaz y Burgos-Ocasio, 2012; Anderson, Shibuya, Ihori, Swing, Bushman, Sakamoto y Barlett, 2010; Bushman y Anderson, 2009; Griffith, 1999; Dietz, 1998; Anderson y Morrow, 1995; Funk y Bushman, 1996; Keepers, 1990; Anderson y Ford, 1986; Cooper y Mackie, 1986). Otro factor identificado en el desarrollo de actitudes y conducta violenta lo es el género, demostrándose que el género masculino tiende a presentar actitudes más favorables a la violencia (Pinilla-Díaz y Burgos-Ocasio, 2012; Posada y Parales, 2012; Moreno, Jesús, Murgui y Martínez, 2012; Feder, Levant y Dean, 2010; Gelles, 2007; Salas y Pujol, 2001; Garbarino, 1999; Kaufman, 1989). Investigaciones recientes señalan, además, que los miembros de la generación $Y$, mejor conocida como milenio, han estado más expuestos a la violencia, a través de los medios (Bosco, 2004; Gorman-Smith, Henry y Tolan, 2004; Krahe, Moller, Huesmann, Kirwil, Felber y Berguer, 2010), lo que los hace que interpreten la violencia de forma muy diferente a generaciones anteriores. Por consecuencia, la justifican según el contexto en el cual esta se presenta, alejándose de los estándares morales que vislumbran la violencia como un acto extremo e injustificado (Pinilla-Díaz levels of tolerance, when compared between men ( $\mathrm{M}$ $=27,522, \mathrm{SD}=22,520)$, and women, $(\mathrm{M}=22,580, \mathrm{DE}=$ $3,441$ ) [t $(71)=3,796, p=.001]$. In fact, the magnitude of the differences, in average, was a large one (Eta squared = .17). In another aspect, the levels of attitude and tolerance amongst women showed differences. The implications of exposure to violence, gender, and the millennial generation are discussed in addition to the need to conduct research among these variables and moral disconnection.

\section{Keywords}

Violence-statistics, violence in education, violence against women, crimes against women, behavior (psychology), social problems, social psychology.

y Burgos-Ocasio, 2012). A esto se le ha llamado desconexión moral (Bandura, 1986; 2016).

\section{Desconexión moral}

Bandura (1986) desarrolló una teoría para explicar los mecanismos subyacentes a los actos que van en contra de los valores promulgados por la sociedad. Esta teoría establece que, a lo largo de sus vidas, los seres humanos establecen un conjunto de valores que rigen sus actos morales; pero, en ocasiones, usan artificios de pensamiento para permitirse la violación de esos valores. Bandura (1986, 2016) le llamó, a este proceso, desconexión moral. Además, identificó los siguientes mecanismos asociados con esta: 1. justificación moral, 2. etiquetamiento eufemístico, 3. comparación ventajosa, 4. desplazamiento de la responsabilidad, 5. difusión de la responsabilidad, 6. distorsión de las consecuencias, 7. deshumanización, y 8. atribución de culpas. También indican que existen dos dimensiones que explican la conducta violenta en los seres humanos: la sociocognitiva, que puntualiza la intencionalidad; y la afectivo-impulsiva, relacionada con la pérdida de control conductual durante la respuesta violenta (Ortega, Sánchez y Menecini, 2002). Estos mecanismos de desconexión moral parecen responder más a los factores sociocognitivos, demostrando con ello la naturaleza cognitiva de justificar la violencia (Caprara, Scabini, Barbaranelli, Pastorelli, Regalia, \& Bandura, 1998). Por medio de estos mecanismos, se ha explicado el uso de la violencia desde la infancia (Martínez- 
González, Robles-Haydar, Amar-Amar, y Crespo-Romero, 2016), desde la milicia (Bandura, 2006) hasta los más terribles actos de terrorismo (Bandura, 1999).

\section{Estudios sobre exposición a la violencia}

Los efectos de la exposición a la violencia, por medio de imágenes, tanto en el cine, la televisión y los videojuegos, han sido resaltados, pero las conclusiones tienden a ser contradictorias. Por un lado, investigadores apuntan a que la exposición a la violencia se traduce en cogniciones, emociones y conductas violentas (Anderson y Bushman, 2001; Anderson y Dill, 2000). Por el otro, se señala que no hay relación directa entre exposición y expresión de conductas violentas (Williams y Skovic, 2005). Al no contarse con estudios concluyentes que establezcan una relación causal entre la exposición a medios violentos y las respuestas agresivas, se ha limitado la legislación y, por ende, las acciones proactivas que limiten o controlen la producción y difusión de material violento por los medios de comunicación (Azar, 2010). No obstante, parece haber un entendido general de que la exposición a material violento produce desensibilización psicológica y apatía o disminución de conductas prosociales para ayudar a las víctimas (Anderson y Ford, 1986; Anderson y Morrow, 1995; Cooper y Mackie, 1986; Dietz, 1998; Funk y Bushman, 1996; Griffith, 1999; Keepers, 1990; Bushman y Anderson, 2009; Anderson et al., 2010). Se entiende, por tanto, que la exposición a material violento por medios de comunicación y tecnológicos constituye un factor de riesgo para el desarrollo de actitudes y tolerancia hacia la violencia.

En una conferencia presentada por Kutner y Olson (2008), en la convención de la American Psychological Association (APA), se expresó la preocupación sobre la influencia en el desarrollo de los niños de las gráficas violentas de los juegos de video, donde se mata sin compasión a policías, civiles, etc. Según estos dos profesionales, los videojuegos son la normativa para los adolescentes y adultos jóvenes, principalmente los varones, quienes juegan más en grupos que las niñas. No obstante, la preocupación reside en cuánto esta exposición influye en la percepción de violencia. Los resultados de las investigaciones reseñadas por estos autores indican que se han encontrado diferencias significativas en cuanto a la excitación violenta, a corto plazo, que esta produce, pero no se ha encontrado correlación en cuanto a incurrir en delitos.
Desde el punto de vista de los factores ambientales (familia, comunidad y sociedad), se ha postulado que la exposición a ambientes violentos representa factores de riesgo para los seres humanos a nivel social y psicológico. Entre estos, se destacan ambientes familiares, escolares, laborales o comunitarios. Como resultado, se crea tolerancia y aceptación hacia la violencia, mediante mecanismos de omisión, permiso, promoción y excusa de esta (AlonsoZayas 2015; Morales, 2005; Reyes, Colón y Moscoso, 2009; ONU, 2010).

En un estudio realizado por Pinilla-Díaz y Burgos-Ocasio (2012) con estudiantes universitarios, que fueron divididos por su cohorte de nacimiento, se encontró que aquellos estudiantes identificados bajo la cohorte correspondiente a la generación del milenio (generación $\mathrm{Y}$ ), presentaron niveles altos de actitud y tolerancia moderada a la violencia. Se observó, además, en este estudio, que los varones presentaban estas características mucho más marcadas que las féminas.

Otro estudio, de corte cualitativo fue realizado por estas autoras como seguimiento a la investigación del 2012. El mismo incluyó estudiantes del nivel avanzado de programas de maestría en Trabajo Social Clínico y doctorado en Psicología Clínica. Este reveló, mediante el análisis de discurso, la presencia de violencia en el entorno, malestar generalizado ante los problemas sociales y actitudes de apoyo y tolerancia a la violencia, en la generación del milenio. Presentando la noción de la violencia como un medio legítimo para solucionar los problemas (Pinilla-Díaz y Burgos-Ocasio, 2013). Las razones que ofrecieron las estudiantes que participaron de esta investigación fluctuó entre exposición a la violencia; violencia como parte de la cotidianidad (videojuegos, películas y otros), dado que lo que "vende" es la violencia; legitimación moral de la violencia como método de solución de problemas y presión de grupo, entre otros.

Violencia, educación y género.

El Departamento de Salud y Servicios Humanos de E.E.UU. (2001) identificó la educación, entre otros, como factor de protección específico hacia la violencia. No obstante, las investigaciones de Pinilla-Díaz y Burgos-Ocasio (2012, 2013), realizadas con estudiantes universitarios pertenecientes a la generación milenio, desde el nivel de subgraduado hasta 
el de graduado y con profesionales en el ejercicio de la profesión, reflejaron que la educación no tuvo ningún efecto para la adopción de actitudes favorables a la violencia y mayor tolerancia a esta.

Lucca-Rodríguez (2015), replicando el estudio de PinillaDíaz y Burgos-Ocasio (2012), esta vez con profesionales de ayuda en las escuelas, no encontró diferencias en actitud, percepción y tolerancia a la violencia al compararlos por generación ( $\mathrm{X}$ y milenio), género y profesión (trabajadores sociales, consejeros y psicólogos). Esto debido a que todos presentaron actitudes altamente favorables hacia la violencia; una percepción o discriminación clara de lo que es violento y lo que no y gran aceptación o tolerancia de esta. Nuevamente, se encuentran estas mismas actitudes. Más aún, la educación, identificada como un factor de protección, tampoco tuvo ninguna influencia, por lo que, en este caso, profesionales del área de la salud mental parecen estar inmersos en la perpetuación del discurso violento, promoviendo actitudes y tolerancia a conductas violentas.

Finalmente, el pertenecer al género masculino se ha identificado como uno de los factores de riesgo a la conducta violenta (Kaufman, 1989; Garbarino, 1999; Salas y Pujol, 2001; Feder, Levant y Dean, 2010; Gelles, 2007; GiménezGarcía, Ballester-Arnal, Gil Llario, Castro-Calvo, y Díaz Rodríguez, 2014). Los resultados de Pinilla-Díaz y BurgosOcasio (2012) y de Negrón-Negrón (2015) parecen confirmar esta visión, no obstante, en cuanto a actitudes y tolerancia a la violencia, los resultados de Lucca-Rodríguez (2015) los contradicen. Una investigación reciente, encontró que las actitudes del papel de género tradicional se asociaron con mayor riesgo para la perpetración de violencia entre los jóvenes que reportaron alta aceptación de violencia (Reyes, Foshee, Niolon, Reidy, Hall, 2016). Ninguno de estos estudios ha explorado exclusivamente las actitudes, la percepción y tolerancia a la violencia en jóvenes milenio.

\section{Generación milenio}

La idea de explorar diferencias en posturas y modos de ver la vida, partiendo de la cohorte de nacimiento de las personas, surge de las teorías de Karl Mannheim, 1927 y de Strauss y Howe (1991). Estos autores, aunque difieren en su estructura, coinciden en establecer que una generación no crece independiente de las influencias externas; y ambas teorías establecen relación de causa y efecto para ilustrar el surgimiento de las características generacionales que las definen. La teoría de Mannheim (1927), reconoce que cada generación influencia y es influenciada por aquellos que le precedieron. Pero también se enfoca en que los grandes eventos históricos cambian a la sociedad. Por tal razón, las personas se asemejan más a sus tiempos que a sus padres. Por otro lado, la teoría de Howe y Strauss (1991) enfatiza la idea de que se dan ciclos de nacimiento y que cada nueva generación presenta una respuesta o reacción diferente a la generación anterior. Estos últimos, acuñaron unos términos para explicar cada generación. Dos de las generaciones que actualmente son más influyentes en las tendencias sociales son la generación $\mathrm{X}$ y la generación milenio. Específicamente, a esta última la constituyen personas nacidas a partir del año 1982 hasta el 2000. Otros nombres para esta generación lo son generation why, o internet generation. Se le considera la generación más grande (después de los baby boomers) y única, ya que incluye el subconjunto de los pioneros de internet. Son la última generación nacida entre el siglo XX y primera de principios del XI. A la generación milenio, por otra parte, también se le conoce por su efecto bumerán, ya que quienes la forman son los que han tenido que volver a casa de sus padres y están retrasando la formación de un hogar por la situación económica actual, la dificultad para encontrar un empleo y para acceder a una vivienda. Entre sus características principales están ser egocentristas, no tener sentido de pertenencia, ser demandantes y conflictivos, cuestionar todo; su vida depende y gira alrededor de la tecnología; no tener apego a las normas sociales y presentar una actitud desafiante y retadora (Robertson, 2007, Strauss y Howe, 2000).

La diferencia en actitudes entre generaciones ha sido ampliamente estudiada en diferentes campos (Robertson, 2007; Shaul, 2007), pero no así en lo relacionado con la percepción de, y la tendencia a, la violencia (Elías, 2003, Bosco, 2004). Dado que en el mundo cada vez se manifiesta más la violencia como solución a los conflictos, se entiende necesario que sea evaluada e intervenida, enmarcándola por las tendencias sociales y morales que dicta la generación en que se nace. El objetivo del presente estudio fue analizar si existen diferencias estadísticamente significativas en las actitudes, la percepción y tolerancia hacia la violencia en estudiantes universitarios de la generación milenio, comparándolas por género. 
Metodología

\section{Participantes}

Participaron en este estudio 78 estudiantes universitarios, del nivel subgraduado, seleccionados por disponibilidad de todos los colegios, concentraciones y especialidades que componen una universidad pública del oeste de la isla de Puerto Rico. Como criterio para la selección de los participantes por generación, se determinó como elegible todo estudiante cuya fecha de nacimiento fluctuara entre el año 1982 y el 2000, siguiendo la categoría identificada por Strauss y Howe, (1991), para la generación milenio. En cuanto al género, 33,3\% (26) eran del género masculino y $66,7 \%$ (52) eran del femenino. La distribución por áreas 0 Facultad de estudio fue de $68,4 \%$ (54), de la Facultad de Ciencias; $11,4 \%$ (9), de la Facultad de Artes y Humanidades; $10,1 \%$ (8), de la Facultad de Ingeniería; $5,1 \%$ (4) de la Facultad Administración de Empresas, y los restantes 5 \% (5) pertenecían a la categoría de otros (no definida su Facultad de estudios).

\section{Instrumento}

Se utilizó la Escala de Actitudes, Percepción y Tolerancia a la Violencia, validada en estudios anteriores, que consiste en 60 afirmaciones que miden Actitud, definida como predisposición o sentimiento favorable (positivo) o desfavorable (negativo) hacia la violencia; Percepción se refiere a la capacidad de reconocer, observar y discriminar la conducta violenta; y Tolerancia es la aceptación de acciones, creencias y costumbres hacia la violencia, sin signos de estrés (VandenBos, 2007).

La validez del instrumento fue determinada por un panel de 10 jueces. El Índice de Validez de Contenido (IVC) fue de .90. La escala fue validada con una muestra de 778 estudiantes universitarios por medio de análisis factorial exploratorio. En la escala de Actitud, el KMO fue de .944; en la escala de Percepción se obtuvo un KMO de .980; y la escala de Tolerancia presentó un KMO de .868. En el análisis factorial, se identificó un solo factor para cada subescala, por lo que la estructura del instrumento, formado por tres factores independientes, fue establecida. El índice de confiabilidad medida por Alpha de Cronbach fue de .930 para la Escala total; y cada una de las escalas obtuvo: Actitud (.897), Percepción (.984) y Tolerancia (.760).

\section{Procedimiento*}

Se diseñó un estudio exploratorio en el que se comparó si existen diferencias significativas por género entre estos jóvenes milenio, en su actitud, percepción y tolerancia hacia la violencia. Para esto, se visitaron los salones de clase en cada Facultad, donde, luego de obtener el consentimiento de aquellos estudiantes dispuestos a participar, se les administró la Escala de Actitudes, Percepción y Tolerancia a la Violencia.

\section{Resultados}

Se realizaron estadísticas descriptivas para los datos sociodemográficos y se hizo un análisis de diferencias entre grupos, por género con $t$ de Student, para las variables dependientes: actitud, percepción y tolerancia a la violencia. En la variable actitud hacia la violencia, $\mathrm{t}(75)=\mathrm{t}-3.758$, $p=.001$., se encontró que no hubo diferencias significativas entre hombres $(M=53.789, D . E .=6.115)$ y mujeres $(M=$ $59.590, D . E .=4.271)$. Esto se reportó también, la variable percepción a la violencia, no reflejó diferencias significativas entre hombres $(M=79.083, D . E .=23.916)$, y mujeres, $(M=$ $88.667, D . E .=19.598), \mathrm{t}(70)=-1.698, p=.097$. Finalmente, se encontraron diferencias significativas en los niveles de tolerancia, al compararlos entre hombre, $(M=27.522, D . E .=$ $22.520)$, y mujeres, $(M=22.580, D . E .=3.441), \mathrm{t}(71)=3.796$, $p=.001$. La magnitud de las diferencias en promedios fue una grande (eta cuadrado $=.17$ )

\section{Discusión}

Analizados los datos, se presenta que no hubo diferencias estadísticamente significativas entre hombres y mujeres en la actitud y percepción hacia la violencia. Las diferencias no significativas encontradas, por género, en estas variables señalan que los participantes (todos perteneciente a la generación milenio) tienen una noción favorable hacia lo que representa la violencia y el rechazo cognitivo (percepción) hacia esta. Este hallazgo es comparable con lo que presentan Pinilla-Díaz y Burgos-Ocasio (2012). En ese estudio, ambos géneros presentan un nivel similar (moderado) al favorecer la violencia. En contraste, en el presente estudio, las mujeres presentan un nivel más alto que los hombres en términos de favorecer la violencia. Esto contrasta significativamente con lo presentado por la literatura (Pinilla-Díaz y BurgosOcasio, 2012; Posada y Parales, 2012; Moreno, Jesús, Murgui 
y Martínez, 2012; Feder, Levant y Dean, 2010; Gelles, 2007; Salas y Pujol, 2001; Garbarino, 1999; Kaufman, 1989) donde se expone que el género masculino tiende a presentar actitudes más favorables a la violencia.

Este hallazgo también se presenta en la variable percepción: ambos géneros reportaron tener altos y claros niveles de percepción hacia violencia; mostrando ambos una clara capacidad de discriminación de lo que es violento y lo que no. Estos hallazgos son similares a lo presentado por PinillaDíaz y Burgos-Ocasio (2012), sin embargo, en el presente estudio, las mujeres presentaron puntajes significativamente más altos en este renglón, entendiéndose que las mujeres tienen mayor claridad de lo que es violencia.

Por otra parte, esto no fue replicado en términos de la tolerancia. En esta variable, los hombres muestran una tolerancia más favorable hacia la violencia que las mujeres. Los puntajes en el presente estudio, al igual que en el de Pinilla-Díaz y Burgos-Ocasio (2012), reflejan puntajes altos para los hombres y moderados para las mujeres. Esto representa que los varones muestran una posición de mayor aceptación de los actos de violencia que las mujeres. Los hallazgos de la presente investigación, especialmente los cambios en la actitud en las mujeres, presentan la posibilidad de la presencia de desconexión moral en la generación milenio (Bandura, 1986, 2016). Ambos géneros, aunque difieren en magnitud, presentan aceptar la violencia. Esto hace concluir que el individuo utiliza mecanismos, de manera inconsciente, para hacer socialmente aceptable la violencia (Reich, 1990). Estos mecanismos son útiles y prácticos, particularmente si se ha estado expuesto consistentemente a esta, creando así una adaptación que permite que los actos de violencia se manejen de manera opuesta a lo que socialmente es permitido. Al verlo como algo rutinario y como instrumento para enfrentar situaciones del diario vivir o para manejar emociones, se interpreta como aceptable. En los hombres se presenta una gran dicotomía: se entiende la violencia como un elemento negativo, pero se tolera. Este hallazgo, presenta la preocupación de los efectos que emergen mediante la exposición constante a la violencia (mediática, familiar, laboral, institucional, entre otras) y el hecho de que está desensibilizando una porción significativa de la sociedad, y que provoca una disminución del afecto negativo ante la violencia real (Fanti, Vanman, Hendrick y Avraamides, 2009).
En conclusión, como factor de riesgo identificado en este estudio para promover la violencia está el ser varón y de una generación más joven (milenio). También se identifica el aumento de aceptación de esta en el género femenino por esta cohorte de nacimiento . Esta aceptación y promoción de la violencia se legitimiza mediante el uso de los mecanismos descritos por Bandura (1986), específicamente, la justificación moral (excusar la violencia), la minimización (distorsión de las consecuencias), la difusión de responsabilidad (ignorar o minimizar las consecuencias negativas de la conducta violenta) y la atribución de culpas (desplazar las consecuencias de los actos violentos hacia la víctima). Disminuir factores de riesgo requiere el entender la violencia y su impacto, especialmente en la generación milenio como un fenómeno multifactorial. La violencia se institucionaliza cada día más, reforzándose en las prácticas interpersonales, familiares y sociales. La presión social de una sociedad machista hace que dicha generación se encuentre más expuesta a este fenómeno. Esto se fundamenta, entre otras, en la presencia de estructuras patriarcales de autoridad y ambiente familiar violento. Además, en medios de comunicación que promueven la violencia, especialmente actos de masculinidad y virilidad en películas, series de televisión y videojuegos Estos elementos se desarrollan para y dirigen a una población joven, donde el símbolo de éxito envuelve la violencia excesiva. Por otra parte, están las redes sociales que permiten su uso para agredir y ridiculizar a otros. También, para presentar y observar actos violentos con el propósito de ganar estatus social y fama.

Por tanto, deben realizarse intervenciones multisectoriales para que la violencia no se vuelva rutinaria en la sociedad y así evitar la desconexión moral, especialmente en los hombres de la generación milenio y generaciones más jóvenes que van surgiendo. Por esto, se hace necesario desarrollar campañas de concienciación sobre la exposición a la violencia, de manera que se transforme en una conducta aceptable a una de rechazo. Esto podría realizarse mediante el uso de los medios de comunicación y tecnológicos preferidos por las generaciones más jóvenes, con el fin de que puedan entender las consecuencias finales de legitimar la violencia.

Además, en términos investigativos, es necesario estudiar los patrones conductuales, sociales y psicológicos de las nuevas generaciones, especialmente con respecto a la violencia. 
Se hace necesario explorar los cambios que presentan las mujeres de la generación milenio, especialmente en la actitud y percepción hacia la violencia. Esto con el propósito de desarrollar intervenciones y modelos de prevención generacionalmente sensibles.

\section{Referencias}

Alonso-Zayas, K. (2015). “Violencia de género: Pandemia de la sociedad". Estudios del Desarrollo Social: Cuba y América Latina. 3(2), 87-98.

Anderson, C.A., \& Ford, C.M. (1986). "Effect of the game player: Short-term effects of highly and mildly aggressive video games". Personality \& Social Psychology. 12(4), 390-401.

Anderson, C.A., \& Morrow, M. (1995). “Competitive aggression without interaction: Effects of competitive versus cooperative instructions on aggressive. Behavior in video games". Personality \& Social Psychology Bulletin. 21(10), 1020-1030.

Anderson, C.A., Shibuya, A., Ihori, N., Swing, E.L., Bushman, B.J., Sakamoto, A., \& Barlett, C.P. (2010). "Violent video game effects on aggression, empathy, and prosocial behavior in Eastern and Western countries: a metaanalytic review". Psychological Bulletin. 136(2), 151173. DOI: $10.1037 / \mathrm{a} 0018251$

Anderson, C.A., \& Bushman, B.J. (2001). "Effects of violent video games on aggressive behavior, aggressive cognition, aggressive effects, physiological arousal, and prosocial behavior: A meta-analytic review of the scientific literature". Psychological Science. 12, 353-359.

Anderson, C.A., \& Dill, K.E. (2000). "Video games and aggressive thoughts, feelings, and behavior in the laboratory and life". Journal of Personality and Social Psychology. 78, 772-790

Azar, B. (2010). "Virtual violence". American Psychological Association. 41(11), 28.

Bandura, A. (1986). Social foundations of thought and action: a social cognitive theory. New Jersey: Prentice-Hall.

Bandura, A. (1999). "Moral disengagement in the perpetration of inhumanities". Personality and Social Psychology Review. 3(3), 193-209.

Bandura, A. (2006). "Mechanisms of moral disengagement in support of military force: The impact of Sep. 11". Journal of Social and Clinical Psychology. 25(2), 141-165.
Bandura, A. (2016). Moral Disengagement: how people do harm and live with themselves. New York: Worth Publishers

Bosco, M. (2004). "Brutally real: why 'The Passion' appeals to young people". Commonweal. 131, 9.

Bushman, B.J., \& Anderson, C.A. (2009). “Comfortably numb: desensitizing effects of violent media on helping others". Psychological Science. 20, 273-277

Bushman, B.J., Calvert, S.L., Dredze, M., Jablonski, N.G., Morril, C., Romer, D., ... Webster, D. W. (2016). "Youth violence: what we know and what we need to know". American Psychologist. 71(1), 17-39

Caprara, V., Scabini, E., Barbaranelli, C., Pastorelli, C., Regalia, C., \& Bandura, A. (1998). "Impact of adolescents' perceived self-regulatory efficacy on familial communication and antisocial conduct". European Psychologist. 3, 125-132.

Cooper, J., \& Mackie, D. (1986). "Video games and aggression in children". Journal of Applied Social Psychology. 16(8), 726-744.

Departamento de Salud y Servicios Humanos de los Estados Unidos. (2001). "Youth violence: a report of the Surgeon General". Recuperado de www.ncbi.nlm.nih. gov/pubmed/20669522

Dietz, T.L. (1998). "An examination of violence and gender role portrayals in video games: Implications for gender socialization and aggressive behavior". Sex Roles. 38(5/6), 425-443.

Fanti, K.A., Vanman, E., Hendrick, C.C., \& Avraamides, M.N. (2009). "Desensitization to media violence over a short period". Aggressive Behavior. 35, 170-187.

Feder, J., Levant, R.F. y Dean, J. (2010). "Boys and violence: a gender-informed analysis". Psychology of Violence. 1, 3-12.

Funk, J.B., \& Buchman, D.D. (1996). "Playing violent video and computer games and adolescent self-concept". Journal of Communication. 26(2), 19-32.

Garbarino, J. (1999). Lost boys: why our sons turn violent and how we can save them. New York: Free Press.

Gelles, R.J. (2007). Family violence. En D.J Flannery, A.T. Vazsony \& I.D. Waldman, (Eds.), The Cambridge Handbook of Violent Behavior and Aggression (403417). New York: Cambridge University Press.

Giménez-García, C., Ballester-Arnal, R., Gil Llario, M., CastroCalvo, J., \& Díaz Rodríguez, I. (2014). “Roles de género y agresividad en la adolescencia". International Journal 
of Developmental and Educational Psychology. 1(2), 373-382.

Gorman-Smith, D., Henry, D. B., \& Tolan, P. H. (2004). “Exposure to community violence and violence perpetration: the protective effects of family functioning". Journal of Clinical and Adolescent Psychology. 33(3), 439-449.

Griffith, M. (1999). "Violent video games and aggression: A Review of the literature. Aggression and violent behavior". 4(10), 203-212.

Howe, N., \& Straws, W. (1991). Generations: the history of America's future. 1584 to 2069. New York: William Morrow and Company.

Howe, N., \& Straws, W. (2000). Millenials rising: the next great generation. New York: Vintage.

Kaufman, M. (1989). Hombres: placer, poder y cambio. Santo Domingo: Ediciones Populares Feministas.

Keepers, G.A. (1990). “Case study: pathological preoccupation with video games". Journal of the American Academy of Child and Adolescence. 29(1), 49-50

Krahe, B., Moller, I., Huesmann L.R., Kirwil L., Felber, J., \& Berguer A. (2010). "Desensitization to media violence: links with habitual media violence exposure, and aggressive behavior". Journal of Personality and Social Psychology. 630-646. doi: 10.1037/a0021711

Kutner, L. y Olson, C. (2008). "Humanizing an inhumane world: Grand theft childhood, video games and media violence". Conferencia ofrecida durante la Convención de APA, Boston, MA.

Lucca-Rodríguez, J.A. (2015). "Actitud, percepción y tolerancia a la violencia en un grupo de trabajadores sociales, orientadores y psicólogos que ofrecen servicios profesionales en las escuelas públicas en la región sur del sistema educativo de Puerto Rico". (Tesis doctoral inédita). Pontificia Universidad Católica de Puerto Rico.

Mannheim, K. (1927). "The problem of generations". En P. Kecskementi (Ed.), Karl Mannheim: Essays (276-322). New York: Routledge.

Martínez-González, M.B., Robles-Haydar, C.A., Amar-Amar, J.J. \& Crespo-Romero, F. A. (2016). "Crianza y desconexión moral en infantes: Su relación en una comunidad vulnerable de Barranquilla". Revista Latinoamericana de Ciencias Sociales, Niñez y Juventud. 14(1), 315-330.

McCrindle, M. (2007). Understanding generation $Y$. North Parramatta: Australian Leadership Foundation.

Morales, M. (2005, 12 de noviembre). Impacto de la violencia en los menores. El Nuevo Día. Recuperado de http//:www.endi.com/ipirit.asp
Moreno, D., Jesús, S.N., Murgui, S., \& Martínez, B. (2012). "Un estudio longitudinal de la reputación social no conformista y la violencia en adolescentes desde la perspectiva del género". Psychosocial Intevention. 21, 67-75.

Negrón-Negrón, M.I. (2015). "Relación entre los estilos de crianza percibidos y niveles de tolerancia a la violencia, en una muestra de adolescentes de 15-17 años". (Tesis de maestría inédita). Pontificia Universidad Católica de Puerto Rico.

ONU (2010). "Estudio sobre tolerancia social e institucional a la violencia basada en género en Colombia". Recuperado de http://www.bdigital.unal.edu. co/48804/1/estudiosobretolerancia.pdf

Ortega, R., Sánchez, V. \& Menesini, E. (2002). "Violencia entre iguales y desconexión moral: un análisis transcultural". Psicothema. 14, 37-49

Posada, R., \& Parales C. J. (2012). Violencia y desarrollo social: Más allá de una perspectiva de trauma. Universitas Psychologica. 11(1), 255-267.

Pinilla-Díaz, A.R. \& Burgos-Ocasio, H. (2013). "La violencia como síntoma de la fragmentación social en estudiantes universitarios: Análisis cualitativo de un conversatorio entre estudiantes de Trabajo Social y Psicología". (Artículo inédito.) Colegio de Estudios Graduados en Ciencias de la Conducta y Asuntos de la Comunidad. Pontificia Universidad Católica de Puerto Rico.

Pinilla-Díaz, A.R. y Burgos-Ocasio, H. (2012). "Generational and gender pespective toward violence among students". Presentación en la Conferencia Anual de la American Psychological Association. Orlando, FL.

Reich, W. (1990). "Understanding terrorist behavior: The limits and opportunities of psychological inquiry". En W. Reich (Ed.), Origins of terrorism: Psychologies, ideologies, theologies, states of mind (261-279). New York: Cambridge University Press.

Reyes, J.C., Colón, H., \& Moscoso, M. (2009). "La violencia entre los adolescentes puertorriqueños y sus factores de riesgo y protección". Cuadernos de la Revista Cayey. 3, 43-73.

Reyes, H.L.M., Foshee, V.A., Niolon, P.H., Reidy, D.E., \& Hall, J.E. (2016). "Gender role attitudes and male adolescent dating violence perpetration: normative beliefs as moderators. Journal of Youth and Adolescence". 45(2), 350-360. http://doi.org/10.1007/s10964-015-0278-0

Robertson, B.E. (2007). "Generation's perception towards dress policies". (Tesis doctoral inédita). University of Laverne, California, US. 
Salas, M., \& Pujol, V. (2001). “Violencia masculina: Una mirada desde una perspectiva de género. Contribuciones a las Ciencias Sociales". Recuperado de http://www. eumed.net/rev/cccss/12/sppl.htm

Strauss, W., \& Howe, N. (1991). Generations: The history of America's future. New York: William Morrow \& Company.

Shaul, C. (2007). "The attitudes toward money as a reward system between the age groups corresponding to boomers, generation $x$ and generation y employees". (Tesis doctoral inédita). Marshall Godsmith School of Management, Fresno, US.

VandenBoss, G.R. (Ed.). (2007). APA Dictionary of Psychology. Washington, D. C: American Psychological Association Williams, D. \& Skoric, M. (2005). "Internet fantasy violence: A test of aggression in an online game". Communication Monographs. 72(2), 217-233. 